\title{
Incorporation of New Technologies into Global Pharmacovigilance
}

Vishwani Persaud-Sharma and Shu-Feng Zhou*

Department of Pharmaceutical Sciences, College of Pharmacy, University of South Florida, Tampa, FL33612, USA

\begin{abstract}
There is an increased concern on the safety of established and newly developed drugs with typical use geared towards aid in the life-long battle to rid the world of threatening and ravishing illness. In an attempt to monitor the adverse drug reactions (ADRs) that frequently arise from consumed medications that have killed many patients each year, the practice of pharmacovigilance allows medical communities to assess and regulate the indications of drugs used for medications throughout the clinical treatment process. With regulations provided by the World Health Organization (WHO) and the Food and Drug Administration (FDA) among other governmental surveyors that form the backbone for these pharmacovigilant establishments worldwide, underdeveloped countries can effectively monitor drug ADRs and the contraindications associated with these medications, allowing for correction and future eradication of harmful drugs for enhanced and effective medical treatment that delivers strong efficacy. From a clinical and research aspect, new technologies are emerging that incorporate these newly arising pharmacovigilant practices including the so-called "Omics" approaches and identification of new biomarkers that are associated with the occurrence of rare and idiosyncratic ADRs.
\end{abstract}

Keywords: Pharmacovigilance; Adverse drug reaction; Pharmacogenomics; Drug

\section{Why is Pharmacovigilance Critical for Avoiding Adverse Drug Reactions?}

With the surge of newly established drugs functioning as mainstream treatment for pharmaceutical companies around the world, the efficacy and overall safety of marketable drugs comes into question. To regulate and govern drug efficacy, the adaptation of pharmacovigilance for pharmaceutically derived establishments must be regulated in an effort to provide the best medicinal treatments available with minimal adversity in terms of toxicity and debilitating outcomes such as death. With limited public access to global safety databases and the increased regulatory enforcement leading to patient accountability from pharmaceutical companies who stand by their drugs and tend to blame the consumer, the need for a comprehensive informational system or database is critical [1]. To rectify this problem, the stringent pharmacovigilance technologies have been employed to monitor and regulate the dispersal, effects, and indications of these marketed compounds. From the perspective of the World Health Organization (WHO), pharmacovigilance can be defined as the technologies and methodologies associated with the recognition, elucidation, assessment, and prevention of the adverse drug reactions (ADRs) established by any given drug or medicinally-related illness $[2,3]$.

Specifically, pharmacovigilance works to monitor the safety signals of drugs in terms of their expected effects compared to concomitant product use where practical methods are specific to different organizations $[1,4]$. Safety signalling compilation is gathered from clinical trial data where the positive effects of treatments are noted in comparison to harmful effects which can generate patterns of common trends resulting in a comprehensive list of adversities specific for each marketable drug [1]. Generally, a safety signal can be defined as the of information that is obtained from a unique or multitude source encompassing observations as well as experimental data with the implication of novel, potentially associated, or findings obtained from a new direction in uncharted territory within a medicinal intervention or series of events associated with salubrious or toxic effects. Signal generation is observed by four methods which include (i) spontaneous reporting, (ii) publishing case reports, (iii) cohort studies, and (iv) post-marketed clinical trials [5]. Difficulty in data accrual arises by the demarcation of the exclusion criteria illustrated in clinical trials, the abridged duration of these trials, and the underrepresented patient population of routine clinical treatment following predetermined drug selection [4].

Apart from the global concern surrounding ADRs, pharmacovigilance also monitors other pivotal factors such as standard medicine factors, medical error, the absence of efficacy reports, medicinal use with the absence of indications, case-based reports in terms of acute and chronic poisoning, mortality assessment due to drug-induced illness, and medicinal consumption error among other key concerns [1]. Specifically, the practice of pharmacovigilance functions to (i) enhance patient safety and overall care for medicinal use and invention, (ii) enhance public health and safety for overall use, (iii) deliver effective evaluation of drugs in terms of risk to promote efficacy and cost effectiveness, and (iv) facilitate drug behaviourbased education and public awareness. Collaboratively, functional pharmacovigilance must ensure cooperative citizenry in order to employ successful outcomes where observation must occur on an individual and population-based level necessitating the intertwining of responsibility of the individual and subjectivity in terms of vigilance [6].

Technological mining of clinical ADR data collection is used in the assessment and detection $\mathrm{d}$ by data mining facilitators likes the FDA's Adverse Event Reporting and recently developed technology

*Corresponding author: Shu-Feng Zhou, Department of Pharmaceutical Sciences, College of Pharmacy, University of South Florida, 12901 Bruce B Downs Boulevard, Tampa, Florida 33612, USA, Tel: 813974-6276; Fax: 813 905-9885; E-mail: szhou@health.usf. edu

Received November 22, 2012; Accepted November 23, 2012; Published November 26, 2012

Citation: Persaud-Sharma V, Zhou SF (2013) Incorporation of New Technologies into Global Pharmacovigilance. J Pharmacovigilance 1: e102. doi:10.4172 2329-6887.1000e102

Copyright: (C) 2013 Persaud-Sharma V, et al. This is an open-access article distributed under the terms of the Creative Commons Attribution License, which permits unrestricted use, distribution, and reproduction in any medium, provided the original author and source are credited. 
like the Electronic Health Records that facilitate automated evaluation and ADR record-keeping [7]. Studies that investigate the update of pharmacovigilance techniques serve to improve the overall outcomes of causality investigation as seen in studies conducted by Theophile et al. [8] and other researcher who are looking to improve consensual expert judgment by evaluating and modifying fifty-three drug events from a pharmacovigilance database, ultimately updating the methods that previously exhibited low specificity with overestimations of drug causation. Pharmacovigilance practices for paediatric drug treatment must acknowledge and incorporate the existence of "off-label" prescriptions that employ the methods of spontaneous notification, a method that has been observed to facilitate greater drug safety and attributes to a higher rate of implemented drug safety in children versus that of adults [9].

While pharmacovigilance methods are individualized from different organizations, practices also differ around the world in terms of evaluation methods where each country and union utilizes their own form of regulatory practices. As depicted by Bonneterre et al. [10], the French National Occupational Disease Surveillance and Prevention Network exemplified much disproportionality in the recruitment and detection of signals, an issue that was comparatively analyzed by the many authors. Similarly, other techniques have been employed on a global scale to functionally regulate pharmacovigilance methods around the world.

\section{A Snapshot of the Recently Developed Technologies of Pharmacovigilance}

While studies in the United States and Europe have paved the way for effective pharmacovigilance protocols, various countries around the world have begun to emerge with avant-garde protocols that can provide a great insight into effective unprecedented pharmacovigilance methods. Statistically, countries like sub-Saharan Africa allot approximately US 3.8 to 4.7 billion dollars towards the development of pharmaceuticals including pharmacovigilance expenses [11]. Realistically, not all countries are as fortunate to establish such protocols as reflected by the $41 \%$ of countries that do not have national policies to reflect the pharmacovigilance and medical safety with 13\%attributed to no functional legal policies to support pharmacovigilance protocols [11].

Due to the wide dispersal of drugs used to combat commonly eradicated illnesses once seen in the US that are now rampant in underdeveloped countries such as the Measles or Rubella, the need for pharmacovigilance is substantiated more than ever. Immunosuppressant diseases such as HIV/AIDS lack developed structural lattices that suffice in regulating the medicines used to treat such harmful diseases versus the regulated drugs used to treat widespread cases of malaria that have pharmacovigilance studies that support drug delivery methods and protect patients from severe and life-threatening contraindications. Even countries like the United Kingdom have experienced medical drawbacks with the lack of clearly defined pharmacovigilant objectives with current protocols reflecting obscure methods for decision-making with few to no audit follow up and poor report quality and poor technology feedback [12]. To correct these inconsistencies, UK pharmacovigilance teams have addressed these issues by employing a subset of pharmacovigilance termed pharmacoepidemiology that works to compile the records of ADR patient complaints through data mining [12]. Recent studies have showed major improvements for proactive pharmacovigilance methods in Europe in terms of risk management initiatives, risk minimization, and pharmacoepidemiology development, methods that are backed by the European Network of Centres of Excellence in the Pharmacovigilance and Pharmacoepidemiology in conjunction with the European Medicines Evaluation Agency [13].

Studies conducted in Nepal functionally analyzed the same criteria in terms of developing more effective pharmacovigilance methodologies after the nation joined the International Pharmacovigilance Program in 2007 [14]. The study reflected the need for investigation into the ADRs in a subset of one hundred and thirteen pharmacovigilance based investigations from varying clinical departments where it was concluded that the most common cause of ADRs reflected the consumption of antimicrobials resulting in maculopapular rash that preceded diarrhoea coupled with regurgitation [14]. While there is no legal mandate that commands Nepalese hospitals to report medicinal safety data, many hospitals do communicate ADEs to regionally located pharmacovigilance centres which are then forwarded to national pharmacovigilance centres which make their way to the Uppsala Monitoring Centre in Sweden by record keeping maintained by the local web-based system termed Vigiflow [14].

On the same note, recent reports illustrate preventable ADRs at a prevalence of fourteen percent due to medication error, resulting in the initiation of a Moroccan government collaboration project with the World Alliance for Patient Safety partnering with UMC in an effort to enhance Moroccan pharmacovigilance methodologies with government regulation provided by the Moroccan Pharmacovigilance Centre [15]. Future plans to couple Moroccan pharmacovigilance centres with poison control centres will lead to the directed and enhanced patient safety in terms of enhanced data collection and drug signal detection to better sever the Moroccan medical community [15]. Current pharmacovigilant methods of medication error initiated by the Moroccan Pharmacovigilance Centre facilitates disclosure of ADRs and medication error by providing local clinical settings with patient information in terms of recurrent drug related incidences through the dispersal of monthly information bulletins aimed towards healthcare professionals [15].

Recent experimental observations for French pharmacovigilance methods work towards the clinical characterization of ADRs in terms of drug-drug interactions that are potentially caused by induction or inhibition of human Cytochrome P450 (CYP 450) enzymes in a comparative pharmacovigilance database investigation to uncover the mechanism and role of different CYPs [16]. The methods employed in the experimental process included the extraction of patient records from the Midi-Pyrénées Pharmacovigilance Centre obtained between January and August of the fiscal year 2008 [16]. Findings associated with this study concluded that very few cases of ADEs were associated with CYP induction or inhibition with a rate of occurrence at a ratio of $1: 100$ [16].

The Democratic Republic of the Congo recently employed pharmacovigilance databases to rectify the scarcity of clinical trial-derived data obtained from second stage patients treated with Nifurtimox-eflornithine Combination Therapy (NECT) for Human African Trypanosomiasis (i.e. African sleeping sickness), an illness that is caused by infection of two types of the protozoan parasite Trypanosoma brucei [17]. Methods for this study include the observance and statistical analysis of patient data post medical treatment for comparative investigation with results concluding that eighty-six patients exhibited at least one mild to moderate ADR [17] Conclusions drawn from this study found ADRs to include vomiting, dizziness, headaches, as well as convulsions and NECT to be an 
effective method of first line treatment for both children and adults with lower ADRs observed in child patients [17]. Pharmacovigilance investigation of randomized clinical trials for the Development of Anti-retroviral Therapies in Africa, namely Uganda and Zimbabwe, have also provided sufficient insights into clinical safety data thereby contributing to effective safety methodologies coupled with WHO supporters [11].

While systematic pharmacovigilance protocols contribute valuable information to the investigations of adverse reactions, public awareness and schematics are diminished, instigating the need for alternative methods such as acquired information from a parental perspective [18]. To gain adequate insight into paediatric drug-induced indications, a study conducted in Finland monitored paediatric pharmacotherapy in terms of ADRs from a parental perspective thereby yielding conclusive ADR results attributed to medicinal administration and dosing with the majority of ADRs linked to anti-infective agents [19]. Additionally, studies conducted in the UK founded unique motivators for parental reporting such as the need to resolve feelings of guilt, the need to further public awareness, and provide support for those who have dealt with the effects of ADRs [18].

\section{Identification of New Biomarkers in Pharmacovigilant Practice}

Use of validated biomarkers and identification of new biomarkers can help prevent or minimize the occurrence of ADRs. Biomarkers can improve the predictability of drug development, and increase the value of preventative and therapeutic interventions by targeting individuals with a high probability of benefits and screening out those at high risk of ADRs. Biomarkers can also be used to screen compounds for ADRs before they enter clinical trials, to inform decisions about whether to develop a drug, to monitor the development of toxicity, to forecast adverse events given wider exposure, or to understand the mechanism by which a drug works. Once a new drug gets into the market, monitoring of the biomarkers in patients for $\mathrm{ADE}$ is always needed. So far, a number of drugs have been withdrawn from the market because of safety concerns and intolerable ADRs. By enhancing the ability to assess whether drug candidates are promising early in development, biomarkers could reduce the costs of developing drugs and bringing them to the market, enhance the safety of new drugs, and improve the cost-effectiveness of drugs by targeting treatment to those patients with the best balance of risk and benefit.

Using more and more highly efficient genetic testing approaches, we have identified that some genetic mutations are associated with specific $\mathrm{ADE}$ and thus can serve as pre-warning biomarkers. For example, some mutations of CYP2C9, 2C19, and 2D6 are associated with an increased risk of ADRs [20]. There are more individuals harbouring the CYP2C $9^{*} 1 /{ }^{*}$ genotype among patients with skin reactions to phenytoin compared with non-exposed controls. The CYP2C $9{ }^{\star} 2$ or ${ }^{*} 3$ genotype was associated with increased risk of gastric bleeding episodes after administration of non-steroidal anti-inflammatory drugs such as celecoxib, diclofenac, ibuprofen, indomethacin, and naproxen [21]. Poor metabolizers of CYP2D6 may have greater risk of ADRs such as venous thrombosis and endometrial cancer caused by tamoxifen [22].

For some drugs, the rare occurrence of serious ADRs may require that genetic studies focus on surrogate markers of ADRs [23]. For example, much work has be done to identify gene variants influencing serum creatine kinase level as a measure of statin intolerance and hepatic transaminase for drug-induced hepatotoxicity [24,25]. However, the extent to which genetic determinants of intermediate toxicity will predict the more extreme phenotypes is yet to be determined. Although genetic and clinical studies of drug-induced QT interval prolongation have provided some insight into predictors of life-threatening ventricular arrhythmias due to drug exposure, the findings have primarily highlighted the clinical importance of extremely rare variants of a small number of genes such as hERG, KCNQ1, and NOS1AP $[24,26,27]$.

Biomarkers used in early or late drug development can serve as phenotypic or clinically effective signals indicating potential ADRs or drug resistance/treatment failure. For example, mutations of the human CC Chemokine receptor 5 (CCR5) or gp120 can confer HIV resistance to maraviroc, an antiretroviral CCR5 receptor antagonist [28]. In addition to playing a role as a co-receptor for CCR5 HIV viral binding and cell entry, CCR5 has a role in the trafficking, localization, and differentiation of leukocytes. Panitumumab, a recombinant, fully human monoclonal antibody against EGFR/HER1, is used to treat advanced colorectal cancer [29]. This protein drug is indicated for use in cancer patients with wild-type rather than mutant KRAS status. Point mutations of KRAS are causally associated with the onset of acquired resistance to panitumumab in colorectal cancers [30]. Thus, detection of CCR5, gp120, and KRAS mutations is important to identify suitable subset of patients to whom maraviroc or panitumumab can be used.

\section{Potential Use of Systems Pharmacology in Pharmacovigilance}

The use of "Omics" or systems pharmacology studies have been employed for more than two decades, yet targeted use for ADR prevention and diagnosis have yet to be firmly established. Systems pharmacology employs both experiments and computational analysis of regulatory networks to develop new understanding of drug action and ADRs across multiple scales of complexity ranging from molecular and cellular levels to tissue and organism levels [31]. Systems pharmacology may provide new approaches for drug discovery for complex diseases such as cancer, diabetes and heart diseases. Over the past decade, genomic, proteomic and metabolomic technologies, network analysis, and other high-throughput studies have provided a wealth of information for integrated systems-level analysis of drug action, molecular targets and ADRs [31]. However, the use of "Omics" approaches in pharmacovigilance is not established.

Despite the global prospective pharmacovigilant programs established to provide poorer countries with effective medicines aimed to treat the ninety-percent disease burden, drug companies face heightened governmental pressures as well as uproar from WHO and patient lobby groups who steadfastly demand removal of financial and legal access barriers [32]. Even though campaigning proves to be effective to some level, the absence of regulated drug ADR monitoring programs still leaves more to be desired by patients and clinicians alike, but how do we take a step in the right direction towards providing the world with effective drug treatment? As suggested by Pirmohamed et al. [32], short-term plans need to make effective use of current or future pharmacovigilant protocols where ADR detection is frequency and population or sample size dependent in terms of drug exposure. Ideally, collaborative efforts amongst academic investigators, drug companies, and governmental parties supervising clinical studies should work towards the development of recurrent ADR reporting forms with data transfer to a single, commonly used database. By the formation of a uniformed and consistent monitoring system, organizations like the East African Network can easily monitor treatments for rubella or malaria with regional surveillance systems with the help and support from public health and drug access campaigns, a system that has the 
operational benefit of data conglomeration from many studies with data that builds upon itself over time [32]. The prospective vision for this implementation would mean demographically specific data that can predict and alert clinicians of warning signals before and after treatment regimes. Similarly, the implementation of systems pharmacology on a global scale utilizes the same ideology in that the use of network analysis monitors drug action and side effects in terms of regulatory networks that survey drug targets and disease gene production functions to heightened mechanistic drug knowledge in terms of the whole genome [31]. The benefit of the implementation of systems pharmacology to pharmacovigilance allows clinicians to understand the drug interaction and disease and gene susceptibility relationship allowing the medical community to predict the outcome of treatment regimens faster and more effectively through pattern recognition of nodal commonalities and differences. Additional benefits of systems pharmacology can be attributed to early disease diagnosis with symptoms precision, and preclinical toxicology reports.

In comparison to spontaneous reporting systems that require the need for drug signal detection analysis and fails to efficiently monitor ADRs, the use of active drug surveillance utilizes the identification of prospective safety problems by systematic patient information accrual for targeted populations through clinical trials, patient registration, and databases of medical records, methods which implement the systems pharmacology approach [4]. Effective Prescription Event Monitoring programs that also follow the systems pharmacology approach can also be employed to survey patients who participate in new drug use. Such cost-effective and systematic programs have already demonstrated successful results in Japan and New Zealand. Today, the promise of systems pharmacology in application to pharmacovigilance functions to monitor and characterize ADRs on a global platform in terms of toxicology networks at unique or numerous action site with varying levels of connectivity [33]. Target sites can originate at the genomic level in terms of mRNA and/or protein as well as the mathematical characterization of nodal interconnectivity and discrepancy. The key to successful systems pharmacovigilance requires consistency to facilitate global use. With a common system in place and surveillance by local, regional, governmental, and WHO partnerships, ADR monitoring can be an effective way to alert treat and prevent recurrent carelessness and deliver effective global healthcare for all, rich or poor.

With the wide dispersal of curative medications functioning in an attempt to rid the world of illness, the need for drug safety is at heightened state of need. Operating at a clinical level in terms of investigation into the cause and frequency of given ADRs for the treatment of diseases, the study of pharmacovigilance categorizes such events in terms of indication observed around the world. Countries such as the United States and the United Kingdom have established coherent pharmacovigilant programs that still need additional refinement; however, to some extent these programs for drug safety monitoring and reporting are established and are in full swing despite minor inconsistencies. As a result to the pivotal question arises: how are ADRs monitored around the world especially in underdeveloped countries in which drug dispersal is the most prevalent and frequent as a result of rapidly mutating drug-resistant illnesses? Through the means of signal detection, clinical trial investigation, data mining, and statistical analysis coupled with WHO program development and surveillance among other governmental collaborations, poorer countries now stand tall and move forward in a coherent effort to effectively and actively monitor pharmacovigilant events. With the implementation of systems pharmacology directed towards to systematic pharmacovigilance protocols and biomarker analysis, the effectiveness of monitoring ADRs have the potential to truly deliver superior medical treatment. Likewise, publically communicating the need for such regulated efforts for drug safety through program and course implementation in pharmacy, nursing, and medical schools can help to further root pharmacovigilance practices in the generations of tomorrow. As an immediate remedy, the medical community must strive to establish systems databases to allow for systematic commonality analysis. The use of a single, clear system will allow the progression of pharmacovigilance to reach new global heights illuminating effective protocols for the world to follow. As a whole, the future for structured, logically developed, and universally governed pharmacovigilant programs are bright and can only get better with effective methods paving the way for newly developed drug safety monitoring around the world regardless of economic class.

\section{Conclusions and Future Perspective}

Pharmacovigilance is a very important approach to identify drug toxicities and ensure drug safety use in the world, but with unbalanced implementation in its practice in developing and developed countries. To establish valid and highly effective pharmacovigilant protocols, clinicians, patients, drug producers and regulatory agents must work diligently together to enhance the ADR monitoring systems.

In the future, pharmacovigilance should adopt more validated new technologies to integrate translational science with clinical practice. When traditional approaches are employed in pharmacovigilance, we should keep eyes on those new technologies such as systems pharmacology in the post-genomic era. It is unlikely to avoid all ADRs but at least we can prevent many predictable ADRs that can be well documented using current available research methods.

\section{References}

1. Lu Z (2009) Information technology in pharmacovigilance: Benefits, challenges, and future directions from industry perspectives. Drug Healthc Patient Saf 1 . $35-45$

2. Knottnerus JA, Tugwell $P$ (2012) Ongoing progress in research on drug exposure and adverse events. J Clin Epidemiol 65: 1029-1030.

3. Luteijn JM, White BC, Gunnlaugsdóttir H, Holm F, Kalogeras N, et al. (2012) State of the art in benefit-risk analysis: medicines. Food Chem Toxicol 50: 26 32.

4. Wise L, Parkinson J, Raine J, Breckenridge A (2009) New approaches to drug safety: a pharmacovigilance tool kit. Nat Rev Drug Discov 8: 779-782.

5. Langlitz N (2009) Pharmacovigilance and post-black market surveillance. Soc Stud Sci 39: 395-420.

6. Talbot JC, Nilsson BS (1998) Pharmacovigilance in the pharmaceutical industry Br J Clin Pharmacol 45: 427-431.

7. Vilar S, Harpaz R, Santana L, Uriarte E, Friedman C (2012) Enhancing adverse drug event detection in electronic health records using molecular structure similarity: application to pancreatitis. PLoS One 7: e41471.

8. Théophile $\mathrm{H}$, André $\mathrm{M}$, Arimone $\mathrm{Y}$, Haramburu $\mathrm{F}$, Miremont-Salamé $\mathrm{G}$, et al. (2012) An updated method improved the assessment of adverse drug reaction in routine pharmacovigilance. J Clin Epidemiol 65: 1069-1077.

9. Autret-Leca E, Marchand MS, Cissok H, Beau-Salinas F, Jonville-Bera AP (2012) Pharmacovigilance in children. Arch Pediatr 19: 848-855.

10. Bonneterre V, Bicout DJ, de Gaudemaris R (2012) Application of pharmacovigilance methods in occupational health surveillance: comparison of seven disproportionality metrics. Saf Health Work 3: 92-100.

11. Miller V, Nwokike J, Stergachis A (2012) Pharmacovigilance and global HIV AIDS. Curr Opin HIV AIDS 7: 299-304

12. Edwards IR (2012) An agenda for UK clinical pharmacology: Pharmacovigilance Br J Clin Pharmacol 73: 979-982. 
Citation: Persaud-Sharma V, Zhou SF (2013) Incorporation of New Technologies into Global Pharmacovigilance. J Pharmacovigilance 1: e102. doi:10.4172/2329-6887.1000e102

Page 5 of 5

13. Moore N, Bégaud B (2010) Improving pharmacovigilance in Europe. BMJ 340: c1694.

14. Jha N, Shankar PR, Bajracharya O, Gurung SB, Singh KK (2012) Adverse drug reaction reporting in a pharmacovigilance centre of Nepal. Australas Med J 5: 268-271.

15. Bencheikh RS, Benabdallah G (2009) Medication errors: pharmacovigilance centres in detection and prevention. Br J Clin Pharmacol 67: 687-690.

16. Danton AC, Montastruc F, Sommet A, Durrieu G, Bagheri H, et al. (2012) Importance of cytochrome P450 (CYP450) in adverse drug reactions due to drug-drug interactions: a PharmacoVigilance study in France. Eur J Clin Pharmacol.

17. Alirol E, Shrumpf D, Heradi JA, Riedel A, de Patoul C, et al. (2012) NifurtimoxEflornithine Combination Therapy for Second-Stage Gambiense Human African Trypanosomiasis: Medecins Sans Frontieres Experience in the Democcratic Republic of the Congo. Clin Infect Dis.

18. Arnott J, Hesselgreaves H, Nunn AJ, Peak M, Pirmohamed M, et al. (2012) What can we learn from parents about enhancing participation in pharmacovigilance? $\mathrm{Br} \mathrm{J}$ Clin Pharmacol.

19. Lindell-Osuagwu L, Sepponen K, Farooqui S, Kokki H, Hämeen-Anttila K, et al. (2012) Parental reporting of adverse drug events and other drug-related problems in children in Finland. Eur J Clin Pharmacol.

20. Zhou SF, Liu JP, Chowbay B (2009) Polymorphism of human cytochrome P450 enzymes and its clinical impact. Drug Metab Rev 41: 89-295.

21. Martínez C, Blanco G, Ladero JM, García-Martín E, Taxonera C, et al. (2004) Genetic predisposition to acute gastrointestinal bleeding after NSAIDs use. $\mathrm{Br}$ J Pharmacol 141: 205-208.

22. Beverage JN, Sissung TM, Sion AM, Danesi R, Figg WD (2007) CYP2D6 polymorphisms and the impact on tamoxifen therapy. J Pharm Sci 96: 22242231.
23. Wheeler J, McHale M, Jackson V, Penny M (2007) Assessing theoretical risk and benefit suggested by genetic association studies of CCR5: experience in a drug development programme for maraviroc. Antivir Ther 12: 233-245.

24. Wilke RA, Dolan ME (2011) Genetics and variable drug response. JAMA 306 306-307.

25. Daly AK (2012) Using genome-wide association studies to identify genes important in serious adverse drug reactions. Annu Rev Pharmacol Toxicol 52 21-35.

26. Sirtori CR, Mombelli G, Triolo M, Laaksonen R (2012) Clinical response to statins: Mechanism(s) of variable activity and adverse effects. Ann Med 44: 419-432.

27. Jeyaraj D, Haldar SM, Wan X, McCauley MD, Ripperger JA, et al. (2012 Circadian rhythms govern cardiac repolarization and arrhythmogenesis. Nature 483: 96-99.

28. Myburgh R, Hochfeld WE, Dodgen TM, Ker J, Pepper MS (2012) Cardiovascular pharmacogenetics. Pharmacol Ther 133: 280-290.

29. Keating GM (2010) Panitumumab: a review of its use in metastatic colorecta cancer. Drugs 70: 1059-1078.

30. Misale S, Yaeger R, Hobor S, Scala E, Janakiraman M, et al. (2012) Emergence of KRAS mutations and acquired resistance to anti-EGFR therapy in colorectal cancer. Nature 486: 532-536.

31. Zhao S, lyengar R (2012) Systems pharmacology: network analysis to identify multiscale mechanisms of drug action. Annual review of pharmacology and toxicology 52: 505-521.

32. Pirmohamed M, Atuah KN, Dodoo AN, Winstanley P (2007) Pharmacovigilance in developing countries. BMJ 335: 462.

33. Bai JP, Abernethy DR (2012) Systems pharmacology to predict drug toxicity: integration cross levels of biological organization. Annu Rev Pharmacol Toxicol. 